\title{
Variant of Rett Syndrome and CDKL5 Gene: Clinical and Autonomic Description of 10 Cases
}

\author{
Giorgio Pini ${ }^{1, *}$ Stefania Bigoni ${ }^{1,3, *}$ Ingegerd Witt Engerström ${ }^{4, *}$ Olga Calabrese ${ }^{5}$ Beatrice Felloni ${ }^{1}$ \\ Maria Flora Scusa ${ }^{1}$ Pietro Di Marco ${ }^{1}$ Paolo Borelli² Ubaldo Bonuccelli ${ }^{2}$ Peter O. O. Julu ${ }^{6, *}$ \\ Jytte Bieber Nielsen ${ }^{7}$ Bodil Morin ${ }^{8}$ Stig Hansen ${ }^{9, *}$ Giuseppe Gobbi ${ }^{10}$ Paola Visconti $^{10}$ \\ Maria Pintaudi ${ }^{11}$ Veneselli Edvige ${ }^{11}$ Anna Romanelli ${ }^{12}$ Fabrizio Bianchi ${ }^{12}$ Manuela Casarano ${ }^{13}$ \\ Roberta Battini ${ }^{13}$ Giovanni Cioni ${ }^{13}$ Francesca Ariani ${ }^{14}$ Alessandra Renieri ${ }^{14}$ Alberto Benincasa ${ }^{15}$ \\ Robert S. Delamont ${ }^{16, *}$ Michele Zappella ${ }^{1}$ and ESRRA group ${ }^{17}$
}

${ }^{1}$ Tuscany Rett Centre Versilia Hospital, Lido di Camaiore, Italy

2 Neurological Department, Versilia Hospital, Lido di Camaiore, Italy

${ }^{3}$ Medical Genetic Unit, University Hospital of Ferrara, Italy

${ }^{4}$ Swedish Rett Center, Östersund Hospital, Östersund, Sweden

${ }^{5}$ Medical Genetic Service, ASL Imola, Italy

6 Breakspear Medical Group, Hertfordshire, United Kingdom

${ }^{7}$ The Danish Center for Rett Syndrome, Kennedy Center, Glostrup, Denmark

8 Habilitation Department, Sundsvall Hospital, Sweden

${ }^{9}$ Institute of Neurological Sciences, South Glasgow University Hospitals, Glasgow, United Kingdom

10 UO Neuropsichiatria Infantile, Ospedale Maggiore, Bologna, Italy

11 UO Neuropsichiatria Infantile, Istituto Giannina Gaslini, Genova, Italy

${ }^{12}$ CNR-Italian National Research Council, Pisa, Italy

13 Dipartimento di Neuroscienze dell' Età Evolutiva, IRCCS Stella Maris, Calambrone, Pisa, Italy

${ }^{14}$ Medical Genetics, University, Policlinico Le Scotte, Siena, Italy

15 UO Pediatria, Ospedale Versilia, Viareggio, Italy

16 King's College Hospital NHS Foundation Trust and King's College London - Regional Neuroscience Centre, London, United Kingdom

17 Other ESRRA members are listed fully in the Acknowledgements

*Members of the European Scientific Rett Research Association (ESRRA group) who have contributed to this work
Address for correspondence Giorgio Pini, Director, Tuscany Rett Centre Versilia Hospital, Via Aurelia 335, Lido di Camaiore 55043, Italy (e-mail: g.pini@usl12.toscana.it).

\footnotetext{
Neuropediatrics 2013;44:237-238.
}

The authors regret missing the inclusion of a sentence in the above article of Neuropediatrics, Volume 43, Number 1, p. 42. The addition is given below:

Acknowledgment

This work was also funded by Ministero della Salute (Grant No.

RF-TOS-2008-1225570-Bando malattie rare to AR).

(c) 2013 Georg Thieme Verlag KG Stuttgart · New York
DOI http://dx.doi.org/ 10.1055/s-0033-1343839. ISSN 0174-304X. 